\title{
Sensitivity Analysis of Simulated Backscattered Electron Emission against Electron Dosage of Buried Semiconductor Features
}

\author{
Maseeh Mukhtar ${ }^{1}$ and Brad Thiel ${ }^{1}$ \\ ${ }^{1 .}$ Colleges of Nanoscale Science and Engineering, SUNY Polytechnic Institute, Albany, NY.
}

Existing attempts to develop performance of semiconductor devices entails size reduction along with cutting-edge non-planar architecture. In the fabrication of these devices, the demand for greater precision measurement of the end dimensions of contact holes and deep trenches has risen. Furthermore, each layer's overlay alignment precision has similarly become very significant. Alternatively, SEM overlay is at present under discussion for use in in-device overlay since overlay measurements from dedicated kerf structures often do not match performance in-circuit, enhanced resolution demanding use cases, in addition to a reference metrology. Primary application spaces are measurement of features from multiple mask levels on the same surface and buried features [1].

Modern CD-SEMs are adept at measuring overlay for cases where all features are on the surface. Though, to measure overlay of buried features, high voltage SEM (HV-SEM) is needed. Gate-to-fin and BEOL overlay are important use cases for this technique. A JMONSEL [2] simulation exercise was performed for these two cases using $10 \mathrm{~nm}$ line/space gratings of 5 lines of graduated increase in depth of burial (10 $-50 \mathrm{~nm}$ ). In an effort to probe the backscattered electron behavior of buried features, the backscattered electron data profile is considered as an image stack to further explore sensitivity dependency vs. energy loss. The backscattered electron image stacks were binned in energy loss intervals of $10-50 \mathrm{eV}$. These simulation results were used to calculate the sensitivity measurements of buried features versus electron dosage for an array of electron beam voltages as previously reported [3].

Next, to emulate real image data the electron emission statistics need to be modulated by dose-dependent shot noise and instrumental artifacts i.e., additive white Gaussian noise (AWGN). Since signal data is aggregated on top of background noise, additive white noise is considered in percentage increase of the noise term under the square root in the sensitivity formula (Figure 2 ). In the formula, $\eta_{\text {ref }}$ is the backscatter yield of the background material in question, $\mathrm{C}$ is the contrast between this material and the background, and $\left(\mathrm{I}_{\mathrm{B}} * \tau\right) / \mathrm{e}$ is the electron dosage (where $\mathrm{I}_{\mathrm{B}}=$ beam current, $\tau=$ dwell time, and $\mathrm{e}=$ elementary charge). The resultant imaging data stream is then assessed so as to understand the noise tolerance of the buried feature signature. Results indicate that there may be optimal energy loss windows along with imaging electron dosages for the detection of buried features for a given material set(s) use case.

References:

[1] B. Bunday et al, Metrology, Inspection, and Process Control for Microlithography XXXI, Proc. SPIE 10145 (2017) p. 101450G.

[2] J. S. Villarrubia et al, Ultramicroscopy 154 (2015), p. 15

[3] M. Mukhtar and B. Thiel, Metrology, Inspection, and Process Control for Microlithography XXXII, Proc. of SPIE 8324 (2018) p. 10585. 


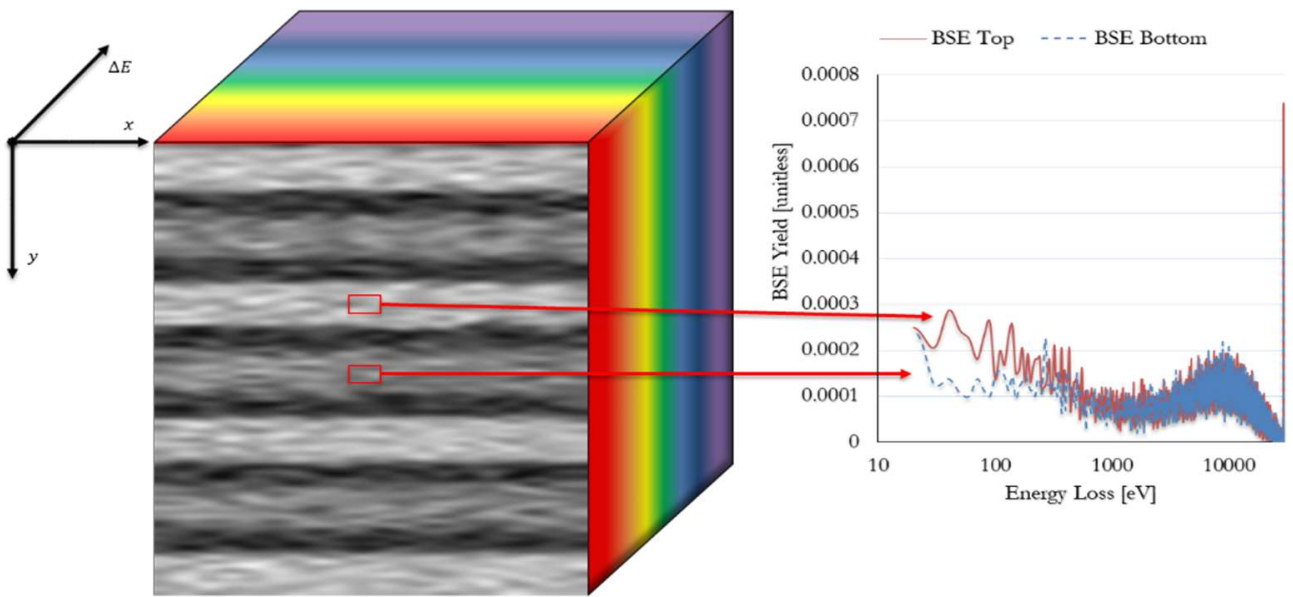

Figure 1. Example simulated energy loss stack profile of buried $\mathrm{Cu}$ gratings filled with $\mathrm{SiO}_{2}$ under $\mathrm{SiO}_{2}$ overlayer.


Figure 2. Left: After shot noise modulation - graphs of backscattered yield vs. energy loss, contrast vs. energy loss, sensitivity vs. energy loss, and sensitivity (using highest contrast value) vs. dwell time (in nanoseconds at $1 \mathrm{nA}$ ) for buried $\mathrm{Cu} / \mathrm{SiO}_{2}$ grating under $\mathrm{SiO}_{2}$ overlayer under $5 \mathrm{keV}$ landing energy. Right: Sensitivity vs. AWGN noise (for $100 \mathrm{~ns}$ dwell time sensitivity results).
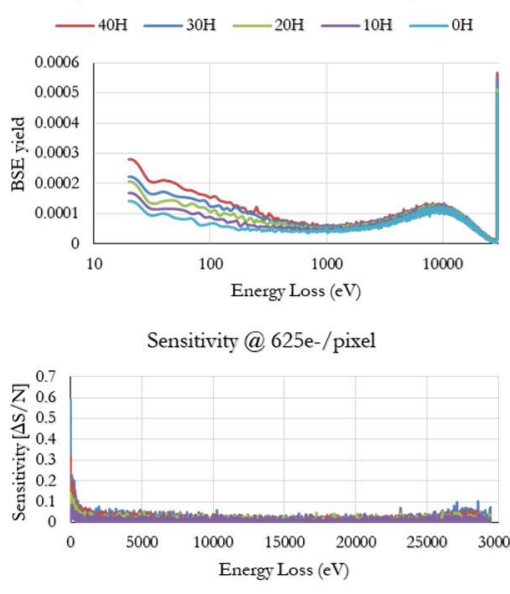

$-10 \mathrm{~nm}-20 \mathrm{~nm}-30 \mathrm{~nm}-40 \mathrm{~nm}$
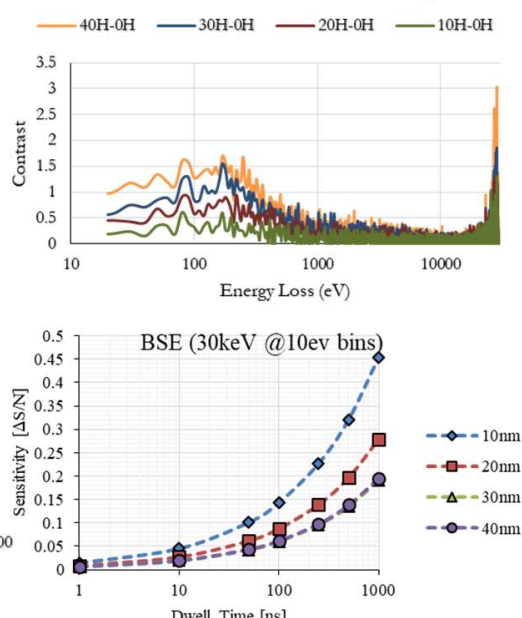

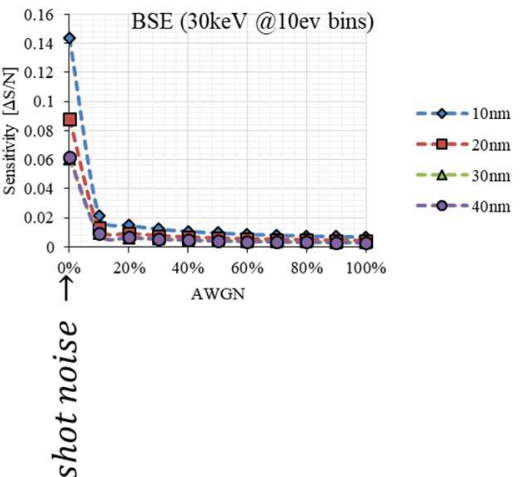

Figure 3. Graphs for buried $\mathrm{Cu} / \mathrm{SiO}_{2}$ grating under $\mathrm{SiO}_{2}$ overlayer under $30 \mathrm{keV}$ landing energy. 